\title{
MicroRNA-210 mediates the hippocampal neurogenesis following traumatic brain injury
}

\author{
Y.-L. Yang ${ }^{1 *}$, T.-C. Huang ${ }^{2}$, Y.-Y.P. Wo ${ }^{1}$, K.-T. Lu ${ }^{2}$ \\ ${ }^{1}$ Department of Biochemical Science and Technology, National Chia-Yi University, Chia-Yi, Taiwan \\ ${ }^{2}$ Department of Life Science, National Taiwan Normal University, Taipei, Taiwan
}

Key words: Mir-210, hippocampus, neurogenesis, traumatic brain injury

Motivation and Aim: Adult neurogenesis is a crucial process for brain tissue repair and remodeling after traumatic brain injury (TBI). MiR-210, a unique and pleiotropic microRNA, has been known to be upregulated in various tissues under hypoxic condition. This study is aimed to investigate the role of miR-210 in TBI-induced neurogenesis and the implicated mechanism.

Methods and Algorithms: TBI was induced by weight-drop device. Immunofluorescence staining of BrdU- and DCX-labeled neurons were used to qualify neurogenesis among different groups after TBI. The expression of miR-210 was evaluated by real-time PCR and the expression of ERK/MEK/Raf cascade was detected by Western blot.

Results: In this study, we found the level of MiR-210 significantly increased within 1 hour after TBI and reached a peak 4 hours after TBI. Administration of a shRNA against miR210 not only reversed the TBI-associated upregulation of miR-210, but also attenuated the TBI-induced neurogenesis. The upregulation of miR-210 after TBI was found to be mediated by HIF- $1 \alpha$ and regulated the phosphorylation of ERK/MEK/Raf cascade, a signal pathway also involved in adult neurogenesis. TBI triggered an approximate 3.0-3.5 fold stimulation in ERK/MEK/Raf phosphorylation, and the administration of miR-210 shRNA effectively dampened the TBI-induced activation of ERK/MEK/Raf cascade.

Conclusions: These results strongly suggest that the increase in miR-210, conferred by TBI, is mediated by HIF-1a expression and might have led to the stimulation of ERK/ MEK/Raf cascade, which in turn promotes the TBI-induced neurogenesis. 